http://jmscr.igmpublication.org/home/

ISSN (e)-2347-176x ISSN (p) 2455-0450

crossref DOI: https://dx.doi.org/10.18535/jmscr/v7i12.108

Journal Of Medical Science And Clinical Research

IGM Publication

An Official Publication of IGM Publication

\title{
Characterization and Biofilm Detection of Candida Species in a Tertiary Care Hospital
}

\author{
Authors \\ Dr Rameez Raja ${ }^{1}$, Dr Beena ${ }^{2}$ \\ ${ }^{1}$ Consultant Microbiologist, Safa Medical Center, Dammam, Saudi Arabia \\ ${ }^{2}$ Professor, Department of Microbiology, MS Ramaiah Medical College, Bangalore
}

\section{Introduction}

Fungal diseases came into clinical importance in the second half of last century. In the past 20 years, the advent of the AIDS epidemic and advances in medical field has even further opened up the clinical mycology field. One of the most frequent opportunistic pathogens among the fungi is the Candida species.

Candida species are ubiquitous yeasts, found as normal commensals on human body surfaces and the gastrointestinal tract. The frequency of Candida infection has increased due to the use of broad-spectrum antibiotics, corticosteroids, immunosuppressive agents, use of invasive medical devices in health care, malignancy etc.

The clinical spectrum of infections caused by them varies from acute or chronic, superficial or deep, localized or systemic. They are recognised as one of the major causes of hospital acquired infections. Although there are about 200 species of Candida, it is well established that only small number are human pathogens. The recent studies suggest that with the introduction of Fluconazole and Itraconazole, there is an increased prevalence of Non-albicans species. Infections with Candida tropicalis, Candida glabrata, Candida krusei and other Candida species are emerging as important opportunistic pathogens. This transition has had a significant clinical impact due to decreased susceptibility of these Non-albicans yeasts to antifungal agents.

Candidastrains possess a number of virulence factors which enable them to spread hematogenously in susceptible hosts and also aid in persistence and colonization of the host tissue. One of the most important factors is the ability to produce biofilms. A significant proportion of Candida species $(73 \%)$ produce biofilms. $^{(1)}$ Biofilm provides protection from environment, increases nutrient availability, metabolic cooperation and acquisition of new genetic traits for the organisms and thus enhanced drug resistance.

In this study, we have isolated the Candida species from different clinical samples. Speciation, antifungal susceptibility pattern and the ability of these isolates to produce biofilm was determined.

\section{Materials and Methods}

94 Candida isolates obtained from different clinical samples coming to Microbiology laboratory from M.S. Ramaiah Teaching Hospital were the source of this study. All non-repetitive isolates of Candida species from the clinical 
samples were included. The samples were urine, blood, Central venous catheter tip, pus, tissue, sputum, body fluids, vaginal swab, Broncheo alveolar lavage fluid.

Candida isolates from these various clinical samples were identified and speculated by Gram stain morphology, Germ tube test, Colour of the colonies on CHROM agar, Dalmau plate culture technique on corn meal agar and Sugar fermentation and Sugar assimilation tests.
Colonies which morphologically resembled yeast on culture plates were subjected to Gram stain Candida appear as gram positive yeast like budding cells, oval to elongated forms of varying sizes, with or without pseudohyphae.

The germ tube test which is used for presumptive identification of Candida albicans and C.dubliniensis was done.

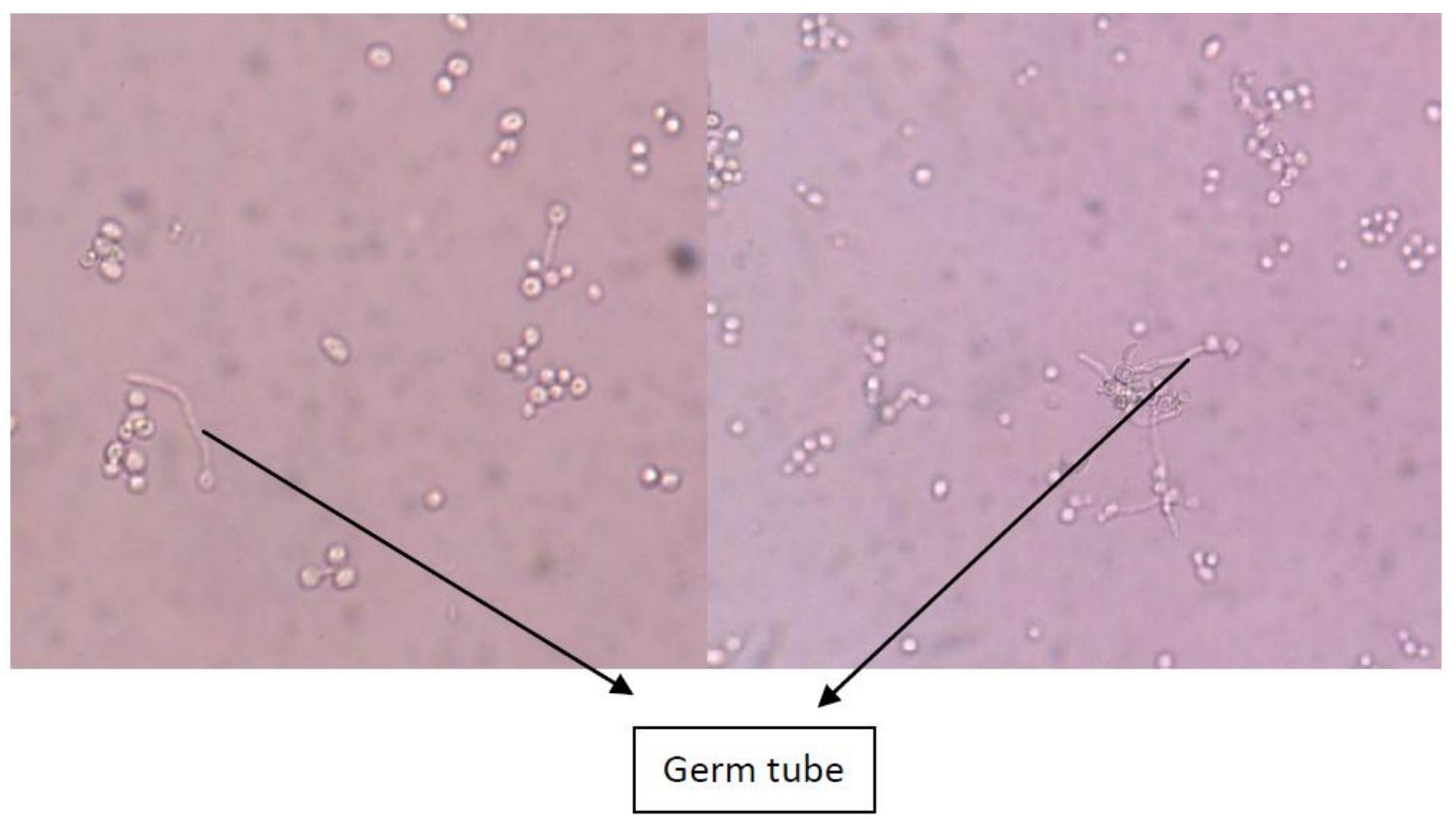

After Gram staining and germ tube test, the isolates were sub-cultured on chromogenic medium (HiCrome candida differential agar; $\mathrm{Hi}$ Media). They were incubated at $35^{\circ} \mathrm{C}$ for 48 hours. All yeast isolates grew well and developed distinctive coloured colonies. Presumptive identification was made by colour and morphology of the colonies as per the manufacturer's instructions. Appearances of Candida species on CHROM agar were as follows: ${ }^{(2,3)}$

\begin{tabular}{|l|l|}
\hline Candida species & Colour of the colonies produced \\
\hline C.albicans & Light green \\
\hline C.tropicalis & Dark blue green centre with pink halo \\
\hline C.krusei & Pink large rough spreading colonies with pale edge. \\
\hline C.guillermondii & Pink to lavender \\
\hline C.parapsilosis & Pale cream coloured colonies. \\
\hline C.glabrata & White to pink, purple \\
\hline C.dubliniensis & Dark green colonies. \\
\hline C.keyfr & Pink to purple \\
\hline C.lusitaneae & Pink to grayish purple. \\
\hline
\end{tabular}




\section{Colours of the colonies on CHROM Agar}
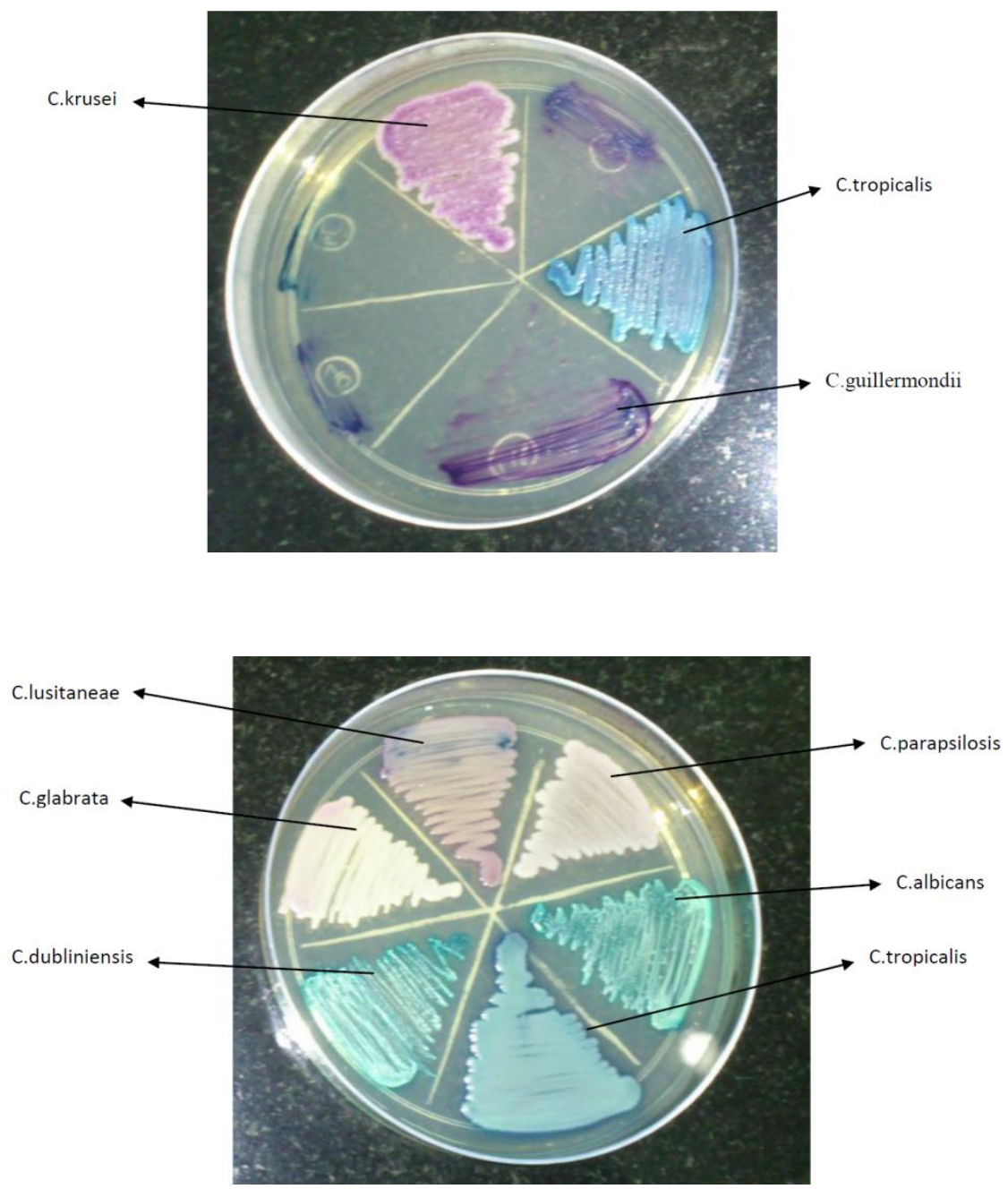

Dalmau plate culture technique. ${ }^{(2,4)}$ Cornmeal agar was used for the production of chlamydospore. The agar was inoculated by making two parallel streaks of young, actively growing yeast colonies. Then it was covered with a 22x22 mm cover slip (sterilized with alcohol and passing over flame). The inoculated medium was incubated at room temperature $\left(25^{\circ} \mathrm{C}\right)$ in dark for
3 days. The plates were then examined. Morphological features like hyphae, pseudohyphae, blastospores, chlamydospores, were noted. Presumptive identification of Candida species was made by correlating the features against the yeast species as follows corresponding to Jagdish Chander Textbook of Medical Mycology ${ }^{(2)}$.

\begin{tabular}{|c|c|}
\hline Terminal chlamydoconidia & C. albicans, C. dubliniensis \\
\hline $\begin{array}{l}\text { Abundant pseudohyphae, pine forest } \\
\text { arrangement, blastoconidia } \\
\text { formed at or between septa }\end{array}$ & C. tropicalis \\
\hline $\begin{array}{l}\text { Elongated yeasts, abundant pseudohyphae } \\
\text { (matchstick-like } \\
\text { appearance) }\end{array}$ & C. krusei \\
\hline Giant hyphae, blastospores at nodes & C. parapsilosis \\
\hline Scant pseudohyphae with chains of blastoconidia & C. guilliermondii \\
\hline Yeasts only & $\begin{array}{l}\text { C. glabrata, C. famata, Pichia } \\
\text { anomala, P. augusta, Cryptococcus neoformans }\end{array}$ \\
\hline $\begin{array}{l}\text { Short, distinctly curved pseudohyphae with } \\
\text { occasional blastoconidia at septa }\end{array}$ & C. lusitaniae \\
\hline
\end{tabular}


Morphology on Dalmau plate technique
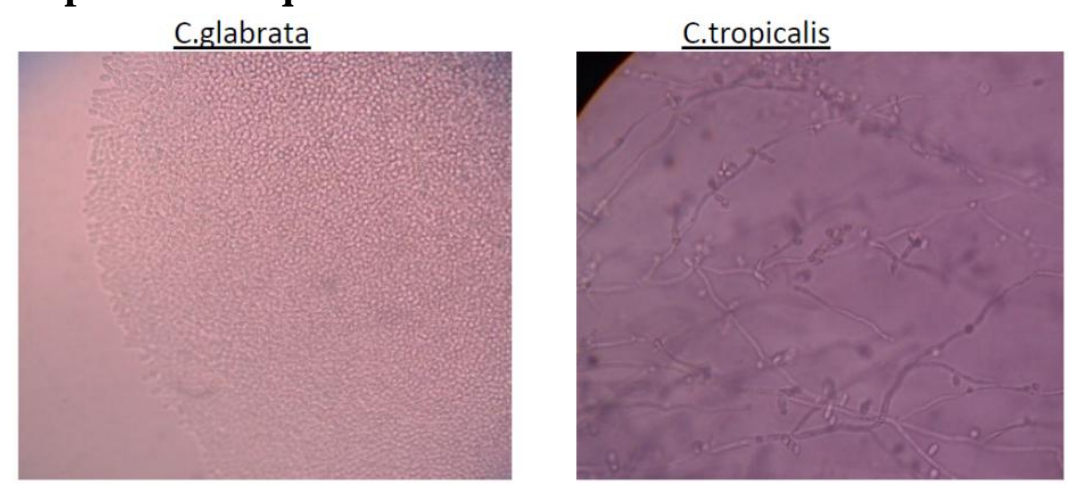

C.albicans

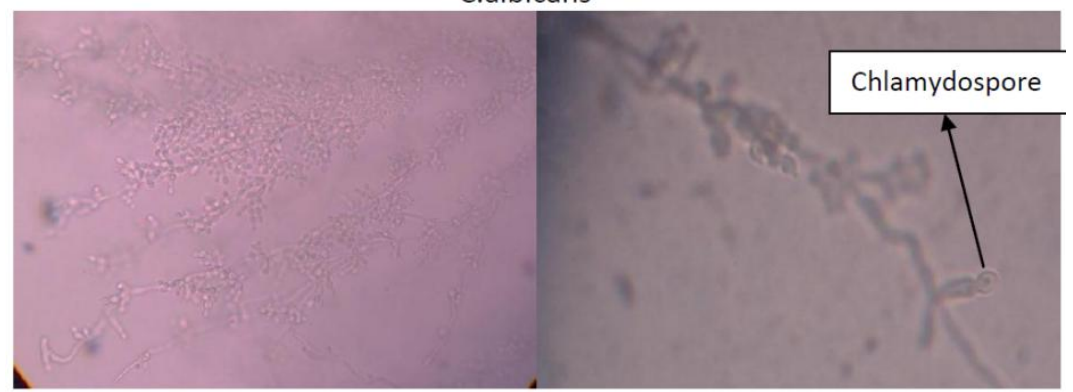

C.parapsilosis

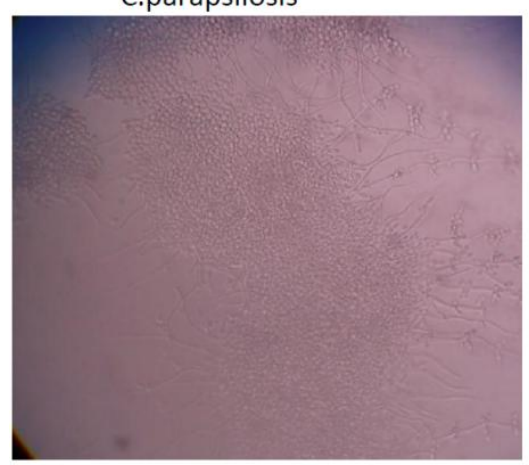

Sugar fermentation and Assimilation. ${ }^{(4)}$ : Heavy growth of the isolates were inoculated onto sugar fermentation tubes containing the appropriate sugars and Durham's tubes. Bromo-thymol blue $(0.025 \%)$ was used as the indicator. The tubes were incubated at $24^{\circ} \mathrm{C}$ up to 1 week. They were examined at 48 hours intervals for acid production (yellow colour) and gas formation (in Durham's tubes). Production of gas indicated fermentation while only acid formation indicated that the sugar has been assimilated. The reactions are read for each sugar separately. The basic sugars used for this test were Glucose, lactose, maltose, sucrose, galactose and trehalose.

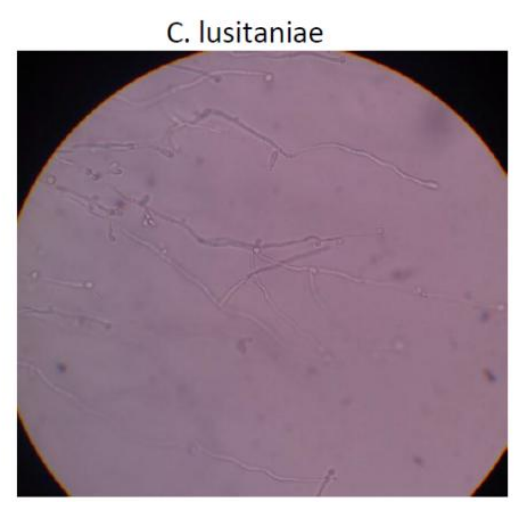

\section{Biofilm Detection}

This was done by Tube method as described by Christensen et al. It is a qualitative assessment of biofilm formation where the microorganisms were grown in Trypticase soy broth with $1 \%$ glucose in test tubes for 24 hours. The tubes were then decanted and washed with PBS (phosphate buffer saline), allowed to dry and stained with crystal violet $(0.1 \%)$. Excess stain from the tubes were then washed with distilled water and the tubes were dried in an inverted position. Biofilm formation was considered positive when a visible stained film, lines the wall and bottom of the tube. $^{(5)}$ 


\section{Biofilm detection by Tube method}
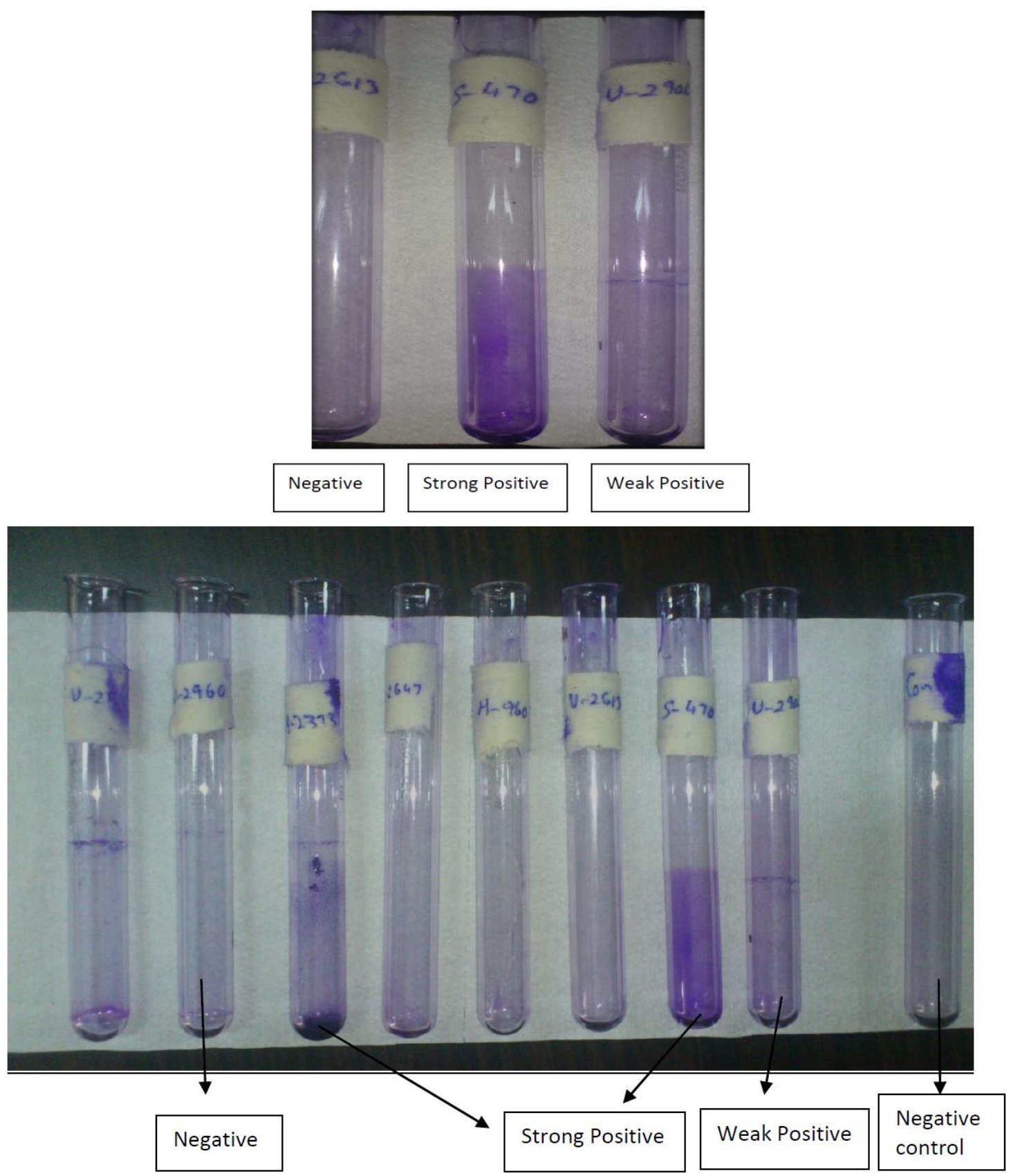

\section{Antifungal Susceptibility Testing: The} antifungal susceptibility test was performed by Kirby Bauer disk diffusion method for Fluconazole (25 mcg/disc), Nystatin (100 U/disc), Amphotericin-B (100 U/disc), Clotrimazole (10 $\mathrm{mcg} / \mathrm{disc}$ ) as per CLSI guidelines 2009. The method described here is only for testing Candida species. ${ }^{(6)}$

Medium: Mueller Hilton agar $+2 \%$ glucose and $0.5 \mu \mathrm{g}$ methylene blue dye.

Turbidity standard for inoculum preparation -0.5 McFarland standard.

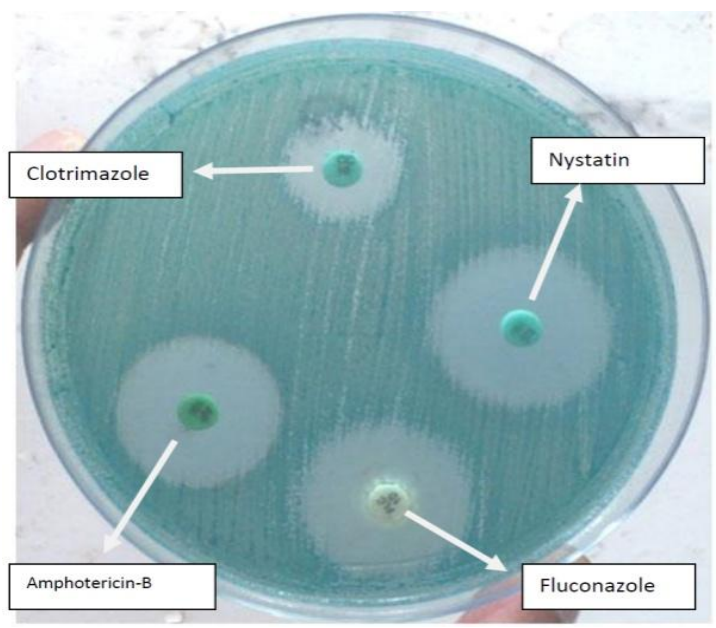




\section{Results}

A total of 94 isolates of Candida species were isolated from different clinical samples during December 2012 to December 2013 received in Microbiology laboratory of M.S. Ramaiah teaching hospital.

The gender and age distribution of these cases were studied and it was found that, out of 94 isolates, $45(48 \%)$ were isolated from male patients and $49(52 \%)$ were isolated from female patients.

The age distribution of the isolates were studied and it was found that, $13.83 \%$ (13/94) were patients in the age group of $<20$ years, $36.17 \%$ (34/94) in $21-40$ years, $30.85 \%$ (29/94) in $41-60$ years and $19.15 \%$ (18/94) in >60 years.

The patient distribution pattern showed that, 90 (95.74\%) patients were In- patients and 4 (4.25\%) were Out patients. Among the In-patients, 22
(23.4\%) were from general wards and 68 (72.34\%) were from ICUs

Among the 94 culture isolates of candida, 45 (47.9\%) were from Urine, 22 (23.4\%) were from blood, $13(13.8 \%)$ were from Central venous catheter tip, $1(1.06 \%)$ was from vaginal swab, 1 $(1.06 \%)$ was from CSF, 6 (6.4\%) were from Pus, $2(2.13 \%)$ were from sputum, $3(3.2 \%)$ were from Broncho Alveolar Lavage (BAL) fluid and 1 $(1.06 \%)$ was from umbilical vein catheter.

\section{Percentage of Candida Species Isolated}

The different species of Candida among the 94 isolates were, C.tropicalis37 (39.36\%) C.albicans 33 (35.11\%), C.glabrata $10 \quad(10.64 \%)$, C.parapsilosis 4(4.26\%) C.lusitaniae 3 (3.19\%), C.guillermondii 3 (3.19\%), C. duliniensis 2 (2.13\%), C.krusei $1(1.06 \%)$ and C.keyfr, 1 $(1.06 \%)$

\begin{tabular}{|l|c|c|c|}
\hline S.No & \multicolumn{1}{|c|}{ Species } & No. of Isolates $(\mathbf{n = 9 4})$ & \multicolumn{1}{c|}{ Percentage (\%) } \\
\hline $\mathbf{1}$ & C.tropicalis & 37 & 39.36 \\
\hline $\mathbf{2}$ & C.albicans & 33 & 35.11 \\
\hline $\mathbf{3}$ & C.glabrata & 10 & 10.64 \\
\hline $\mathbf{4}$ & C.parapsilosis & 4 & 4.26 \\
\hline $\mathbf{5}$ & C.lusitaniae & 3 & 3.19 \\
\hline $\mathbf{6}$ & C.guillermondii & 3 & 3.19 \\
\hline $\mathbf{7}$ & C.dubliniensis & 2 & 2.13 \\
\hline $\mathbf{8}$ & C.krusei & 1 & 1.06 \\
\hline $\mathbf{9}$ & C.keyfr & 1 & 1.06 \\
\hline
\end{tabular}

\section{Biofilm production among the Candida isolates}

Among the 94 isolates of Candida, 34 (36.17\%) of them were found to be biofilm producers by tube method.

\begin{tabular}{|l|c|c|}
\hline S.no & Species & Tube method \\
\hline $\mathbf{1}$ & C.tropicalis (37) & 18 \\
\hline $\mathbf{2}$ & C.albicans (33) & 10 \\
\hline $\mathbf{3}$ & C.glabrata (10) & 0 \\
\hline $\mathbf{4}$ & C.lusitaniae (3) & 2 \\
\hline $\mathbf{5}$ & C.guillermondii (3) & 2 \\
\hline $\mathbf{6}$ & C.krusei (1) & 1 \\
\hline $\mathbf{7}$ & C.parapsilosis (4) & 0 \\
\hline $\mathbf{8}$ & C.keyfr (1) & 1 \\
\hline $\mathbf{9}$ & C.dubliniensis (2) & 0 \\
\hline Total (94) & \multicolumn{2}{|c|}{$34(36.17 \%)$} \\
\hline
\end{tabular}

The ability to form biofilm was most commonly seen in C.tropicalis (19), followed by C.albicans (10). 
Antifungal susceptibility pattern

\begin{tabular}{|l|c|c|c|}
\hline Antifungal agent & \multicolumn{3}{|c|}{ Susceptibility pattern } \\
\hline & Sensitive & Intermediate & Resistant \\
\hline Fluconazole & $49(52.13 \%)$ & $6(6.38 \%)$ & $39(41.49 \%)$ \\
\hline Amphotericin-B & $89(94.68 \%)$ & $2(2.13 \%)$ & $3(3.19 \%)$ \\
\hline Nystatin & $90(95.74 \%)$ & $1(1.06 \%)$ & $3(3.19 \%)$ \\
\hline Clotrimazole & $40(42.55 \%)$ & $10(10.64 \%)$ & $44(46.81 \%)$ \\
\hline
\end{tabular}

Antifungal susceptibility pattern showed that among the 94 Candida isolates, 39 (41.49\%) were resistant to Fluconazole, 3 (3.19\%) were resistant to Amphotericin-B, 3 (3.19\%) were resistant to Nystatin and $44(46.81 \%)$ were resistant to Clotrimazole.

\section{Discussion}

The most common specimen from which the Candida isolates were obtained, was urine (45/94) out of which 29 were catheterized sample. The second most common sample was blood (22/94), followed by Central venous catheter tip (13/94), Pus (6/94), BAL fluid (3/94), sputum (2/94), vaginal swab (1/94), CSF (1/94) and Umbilical vein catheter (1/94). Candida species have a predilection towards causing device associated infections. This can be correlated in our study as many of the isolates $(43 / 94,-45.7 \%)$ were from intravenous and urinary catheters.
In our study, majority of the Candida were isolated from In-patients (95.74\%). Among the Inpatients, samples from ICUs yielded more number of isolates $(72.34 \%)$ compared to the general wards $(23.4 \%)$. This could be attributed to the fact that most of the patients in ICUs are critically ill, are on broad spectrum antibiotics and more often have intravenous or urinary catheters. Also the length of hospital stay is more for ICU patients compared to general wards, which could contribute to the infection caused by candida.

The most common species isolated was C.tropicalis $(39.36 \%)$, followed by C.albicans $(35.11 \%)$. In the recent times, tropical countries like India show predominance of C.tropicalis. This can be correlated in our study. C.albicans (44.4\%) was the most common species causing Candiduria followed by C.tropicalis (40\%). C.albicans $(36.4 \%)$ and C.tropicalis (32\%) predominated in blood samples also. This is comparable to other similar studies as follows.

\section{Summary of other studies regarding species distribution}

\begin{tabular}{|c|c|c|}
\hline Author & Results (Most common) & Publication \\
\hline $\begin{array}{l}\text { A.Chakrabarti, T.C.S } \\
\text { Reddy, and S. singhi. }\end{array}$ & $\begin{array}{c}\text { C. tropicalis }(29.7 \%) \\
\text { C. albicans }(9.9 \%)\end{array}$ & $\begin{array}{l}\text { IJMR, Dec., 1997: 513- } \\
516 .\end{array}$ \\
\hline Kauffman CA,Vazquez JA & $\begin{array}{l}\text { C.albicans }(51.8 \%) \\
\text { C.glabrata }(15.6 \%) \\
\end{array}$ & $\begin{array}{c}\text { Clin.Infect } \\
\text { Dis.2000;30:14-18 } \\
\end{array}$ \\
\hline Rani R,Mahapatra NP & $\begin{array}{l}\text { C. tropicalis }(69.1 \%), \\
\text { C.glagrata }(7.4 \%) \\
\end{array}$ & IJMM, 2002, .20., 42-44 \\
\hline $\begin{array}{l}\text { XistoSenaPassos, } \\
\text { Werther Souza Sales }\end{array}$ & $\begin{array}{c}\text { C.albicans }(69.1 \%) \\
\text { C.glabrata }(7.4 \%)\end{array}$ & $\begin{array}{c}\text { Mem Instoswaldo Cruz, } \\
\text { Rio De Janeiro, Vol } \\
\text { 10018:925-928,Dec } 2005 .\end{array}$ \\
\hline $\begin{array}{l}\text { S.Shivaprakash, } \\
\text { K Radhakrishnan }\end{array}$ & $\begin{array}{l}\text { C. tropicalis }(35.6 \%), \\
\text { C.albicans }(3.4 \%) .\end{array}$ & IJMM, 2007; 25(4):405-7. \\
\hline Vijaya et al & $\begin{array}{l}\text { C. tropicalis }(35.29 \%) \text {, } \\
\text { C.albicans }(45.9 \%) \text {. }\end{array}$ & $\begin{array}{c}\text { Journal of Clinical and } \\
\text { Diagnostic Research. 2011; } \\
\text { 5(4): 755-757 }\end{array}$ \\
\hline In our study & \multicolumn{2}{|c|}{$\begin{array}{l}\text { C.tropicalis }(39.4 \%) \\
\text { C.albicans }(35.1 \%) \\
\end{array}$} \\
\hline
\end{tabular}

A study conducted by Muni et al, showed that biofilm production was seen with $64 \%$ of the isolates. The biofilm production was more with non-albicans Candida spp (78.9\%) than Candida albicans $(54.8 \%){ }^{(7)}$ 
Another study conducted by Vinitha et al., showed that $73 \%$ of Candida species obtained from the clinical specimens produced biofilm. Biofilm production was seen more in $C$. kruseiand $C$. tropicalis. ${ }^{(1)}$

In a study conducted by Dag et al., $38.7 \%$ of the candida species were biofilm positive by Tissue culture plate method and $26.3 \%$ of them were positive by Tube method. ${ }^{(8)}$

In our study, $39.36 \%$ of the candida species were positive for biofilm formation. This high positivity for biofilm formation could be because many of the isolates were from infections associated with intravenous or urinary catheters. It is a known fact that Candida species have the tendency to form biofilm on prosthetic surfaces.

In a study conducted by Vijaya et al., isolates were $100 \%$ sensitive to Amphotericin B, Clotrimazole, Nystatin and Ketaconozole $87.5 \%$ of C.krusei, $36 \%$ C.tropicalis, $6.5 \%$ C.albicans were resistant to Itraconozole. $25 \%$ of C.krusei, $28 \%$ C.tropicalis showed resistant to Fluconazole. C.dubliniensis was resistant only to Itraconozole. $^{(9)}$

In a study by De Luca et al., the rate of susceptibility to Fluconazole was $100 \%$ for C.albicansand C.parapsilosis. Decreased susceptibility to Fluconazole was mostly seen with C.glabrata, which was $76.5 \%$ susceptible in a dose-dependent manner. The echinocandins showed a good performance for C. albicans, and maintained a good MIC distribution in C. $_{\text {glabrata. }}{ }^{(10)}$

In our study, a high number of candida species showed resistance to Fluconazole (41.49\%) and Clotrimazole $(46.81 \%)$. Very few number of isolates showed resistance to Amphotericin-B (3.19\%) and Nystatin (3.19\%). Among the different candida species, resistance to Fluconazole was seen highest in C.glabrata $(70 \%)$ followed by C.tropicalis (43.24\%). Thus, in our study, it is noted that Non-albicans candida show more resistance to Fluconazole compared to candida albicans. Majority of the candida species were susceptible to Amphotericin-B and Nystatin.
Resistance to Fluconazole can be attributed to its increased use in the recent days and enhanced drug resistance mechanisms exhibited by the candida species. Some of the species C.glabrata and C.krusei also exhibit intrinsic resistance to Fluconazole, which can be observed in our study. The growing number of reports of resistance to antifungal drugs presses the need for rapid and precise identification of Candida isolates to species level, test them for biofilm and study their antifungal susceptibility pattern for effective treatment and management strategies. Disk diffusion is simple and easy to perform but to confirm resistance, broth dilution method to find the MIC is recommended. This would also pave way for the judicious use of the antifungal agents.

\section{References}

1. Mohandas V, Ballal M. Distribution of Candida Species in Different Clinical Samples and Their Virulence: Biofilm Formation, Proteinase and Phospholipase Production: A Study on Hospitalized Patients in Southern India. J Glob Infect Dis. 2011;3(1):4-8.

2. Candidiasis Ch 20 In Jagdish Chander Textbook of Medical Mycology, Mehta publishers, New Delhi, 3rd edition, 2010,266-283.

3. Hospenthal DR, Beckius ML, Floyd KL, Horvath LL, Murray CK. Presumptive identification of Candida species other than C. albicans, C. krusei, and C. tropicalis with the chromogenic medium CHROM agar Candida. Ann Clin Microbiol Antimicrob. 2006 Jan 3;5(1):1.

4. WHO, Laboratory manual for diagnosis of fungal opportunistic infections in HIV/AIDS patients; 2010.

5. Christensen GD, Simpson WA, Bisno AL, Beachey EH. Adherence of slimeproducing strains of Staphylococcus epidermidis to smooth surfaces. Infect Immun. 1982 Jul;37(1):318-26. 
6. Clinical and Laboratory Standards Institute. 2009. Method for antifungal disk diffusion susceptibility testing of yeasts; approved guideline, 2nd ed., M44-A2. Clinical and Laboratory Standards Institute, Wayne, PA.

7. Crump JA, Collignon PJ. Intravascular catheter-associated infections. Eur J Clin Microbiol Infect Dis Off Publ Eur Soc Clin Microbiol. 2000 Jan;19(1):1-8.

8. Dag I, Kiraz N, Oz Y. Evaluation of different detection methods of biofilm formation in clinical Candida isolates. Afr J Microbiol Res. 2010 Dec 18;4(24):27638.

9. Vijaya D., Harsha T.R., Nagaratnamma T. "Candida Speciation Using Chrom Agar" Journal of Clinical and Diagnostic Research. 2011; 5(4): 755-757.

10. De Luca C, Guglielminetti M, Ferrario A, Calabr M, Casari E. Candidemia: species involved, virulence factors and antimycotic susceptibility. New Microbiol. 2012 Oct;35(4):459-68. 